\title{
Comparison of different snacking models in post-secondary students in an urban setting
}

\author{
Tay Mia Eng ${ }^{1}$, Chew Yi Chien ${ }^{1}$, Emma Foster ${ }^{2}$ and Iain A Brownlee ${ }^{3}$ \\ ${ }^{1}$ School of Chemical \& Life Sciences, Nanyang Polytechnic, Singapore, \\ ${ }^{2}$ Human Nutrition Research Centre, Newcastle University, NE2 $4 H H$ and \\ ${ }^{3}$ Human Nutrition Research Centre, Newcastle University, Singapore
}

While snacking is likely to impact total dietary intake, the definition of "a snack" remains contentious. It is postulated whether a person perceives an eating occasion to be a meal or a snack may influence the amount one eats and their subsequent intake at the next eating occasion ${ }^{(1)}$. The study uses three previously proposed models to define snacking to compare the contribution towards energy intake from snacks, in post-secondary school students from Singapore. Three models of snacks are derived using a combination of the following criteria: Criterion 1 (C1) - Time of consumption outside of recognized "main meal" times (i.e. breakfast: 0600-1000, lunch: 1200-1400 \& dinner: 1800-2000). Criterion 2 (C2) - Portion size. If the amount of food in one eating occasion falls below $570 \mathrm{~g}$, it is classified as a snack. The value of $570 \mathrm{~g}$ was defined as the estimated portion size to provide a third of the food-based dietary guidelines used in Singapore ${ }^{(2)}$; Criterion 3 (C3) - If eating occasion is less than $15 \%$ of total energy intake, then it is a snack ${ }^{(3)}$. Depicts the proposed models, which were based on eating occasions that met 2 of the above criteria. The defined three models were then applied to an existing set of 24-h recall data collected from 17-21 year old students from Singapore $(n=300)$. Dietary data were analysed using WinDiets ${ }^{\mathrm{TM}}$ software and energy contribution from items defined as snacks using the different models calculated. Statistical analysis was performed using the Statistical Package for Social Sciences, SPSS, version 23 for Windows and statistical significance for all tests was defined at $p$-value $<0 \cdot 05$. One sample t-test was applied to evaluate the congruence between the models.

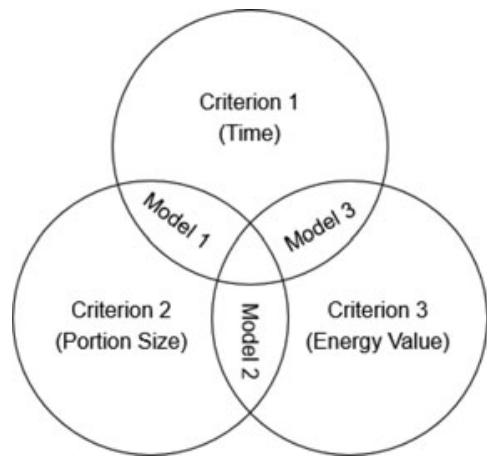

Table 1. Mean total energy (TE) \% contributed from snacks and p-value

\begin{tabular}{lrrr}
\hline & Model 1 & \multicolumn{1}{c}{ Model 2 } & \multicolumn{1}{c}{ Model 3 } \\
\hline Mean TE\% (SD) & $26 \cdot 1(26 \cdot 0)$ & $11 \cdot 1(10 \cdot 7)$ & $5 \cdot 3(7 \cdot 3)$ \\
p-value (vs Model 1) & - & $<0 \cdot 0005$ & $<0 \cdot 0005$ \\
p-value (vs Model 2) & $<0 \cdot 0005$ & - & $<0 \cdot 0005$
\end{tabular}

Fig. 1. Snacking models

There was a significantly different estimate of $\%$ contribution of snacks to daily total energy intake $(\mathrm{p}<0 \cdot 005)$ for all three models. This suggests a need for a consensus definition of what constitutes a snack in order to consider the impact of snacking on total dietary intake.

1. Pliner P, Zec D (2007) Meal schemas during a preload decrease subsequent eating. Appetite 48(3): 278-88.

2. Health Promotion Board S. A Healthy Food Foundation - for Kids and Teens 2017 [cited 201707 February]. Available from: https://www.hpb.gov. sg/HOPPortal/health-article/6086.

3. Murakami K, Livingstone MBE (2016) Decreasing the number of small eating occasions ( $<15 \%$ of total energy intake) regardless of the time of day may be important to improve diet quality but not adiposity: a cross-sectional study in British children and adolescents. British Journal of Nutrition 115(02): $332-41$. 